\title{
When is surgical therapy for head and neck malignant epithelial tumors not indicated?
}

\author{
Carlos Suárez • Robert P. Takes · Carl E. Silver · Juan P. Rodrigo • Primož Strojan • Julia A. Woolgar • \\ Missak Haigentz Jr. • H. Hakan Coskun • Remco de Bree • Randall P. Owen • Mohamed N. Elsheikh • \\ Ashok R. Shaha $\cdot$ Alessandra Rinaldo $\cdot$ Alfio Ferlito
}

Received: 23 December 2013 / Accepted: 16 January 2014 / Published online: 4 February 2014

(C) Springer-Verlag Berlin Heidelberg 2014

The choice of primary treatment of malignant tumors of the head and neck, surgical or non-surgical, depends on factors such as tumor type, site and locoregional extension; the presence of distant metastases; available facilities, personnel and experience of the treating center; and patient factors like comorbidity. Moreover, the choice of treatment modalities is based on the expected optimal oncologic

This paper was written by members of the International Head and Neck Scientific Group (http://www.IHNSG.com).

\section{Suárez · J. P. Rodrigo}

Department of Otolaryngology, Hospital Universitario Central de Asturias, Oviedo, Spain

C. Suárez · J. P. Rodrigo

Instituto Universitario de Oncología del Principado de Asturias, Oviedo, Spain

\section{R. P. Takes}

Department of Otolaryngology-Head and Neck Surgery,

Radboud University Medical Center, Nijmegen, The Netherlands

\section{E. Silver}

Departments of Surgery and Otolaryngology-Head and Neck

Surgery, Albert Einstein College of Medicine,

Montefiore Medical Center, Bronx, NY, USA

\section{P. Strojan}

Department of Radiation Oncology, Institute of Oncology,

Ljubljana, Slovenia

\section{J. A. Woolgar}

Oral Pathology, School of Dentistry, University of Liverpool,

Liverpool, UK

\section{Haigentz Jr.}

Department of Medicine, Division of Oncology,

Albert Einstein College of Medicine, Montefiore Medical Center,

Bronx, NY, USA outcome weighed against the best quality of life and functional outcomes after treatment.

In the interest of optimal patient care, we examine the reasons for recommending non-surgical therapies for certain epithelial cancers of the head and neck. Generally, the usual indications for choice of non-surgical rather than surgical treatment are (1) for certain histological tumor types that respond well to non-surgical treatments, (2) advanced locoregional disease impossible to extirpate completely or without producing an unacceptable functional outcome,

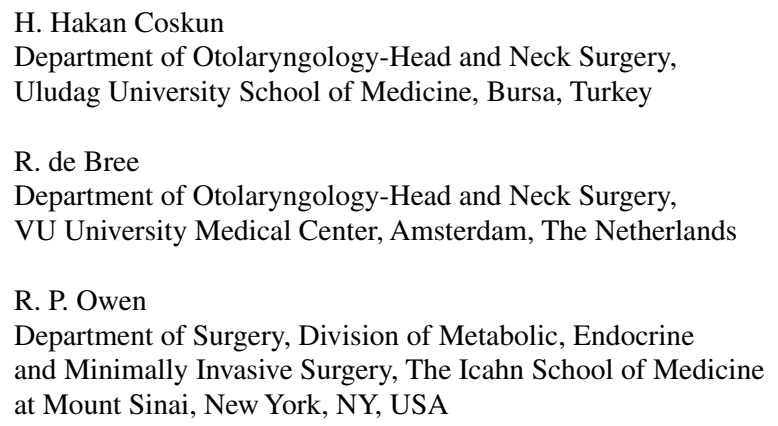

M. N. Elsheikh

Department of Otolaryngology-Head and Neck Surgery, Tanta University, Tanta, Egypt

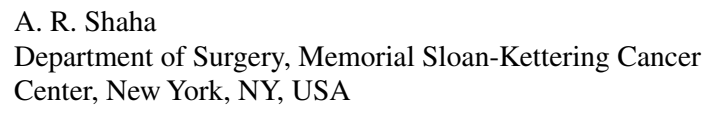

A. Rinaldo $\cdot$ A. Ferlito

ENT Clinic, University of Udine, Udine, Italy

\section{A. Ferlito $(\square)$}

ENT Clinic, University of Udine, Piazzale S. Maria della

Misericordia, 33100 Udine, Italy

e-mail: a.ferlito@uniud.it 
and (3) incurable disease due to patient factors such as severe comorbidity or presence of distant (M1) disease.

Histologic type of the tumor can be the predominate factor in precluding the use of surgical therapy. A range of head and neck malignant epithelial tumors are highly sensitive to radiotherapy which is commonly employed as definitive treatment, alone or in combination with chemotherapy for these histologies. In some cases, varieties of tumor within a given classification will select the indication for surgical or non-surgical treatment. A good example of this is tumors with neuroendocrine differentiation, particularly in the sinonasal area as well as at other sites, such as the larynx.

Sinonasal carcinoma with neuroendocrine differentiation is a rare group of tumors consisting of esthesioneuroblastoma (olfactory neuroblastoma), sinonasal neuroendocrine carcinoma, sinonasal undifferentiated carcinoma and small cell neuroendocrine carcinoma. While the esthesioneuroblastoma may be treated by surgery alone depending on its stage [1], the small cell and undifferentiated varieties are highly aggressive tumors that have usually metastasized regionally and distantly by the time of initial diagnosis. Surgery is generally considered futile for these tumors. Some patients with advanced stage esthesioneuroblastoma or sinonasal undifferentiated carcinoma can benefit from aggressive chemoradiotherapy and radical surgery. Small cell neuroendocrine carcinoma, however, has a very high propensity for distant metastasis, poor prognosis, and often warrants only systemic therapy [2].

Surgery, particularly as initial therapy, is rarely employed as primary treatment of malignant tumors arising in the region of the nasopharynx. This is partly due to complex anatomical relationships among structures in the upper part of pharyngeal axis and just below the base of skull. Nasopharyngeal undifferentiated carcinoma is a distinct clinico-pathologic entity with a strong etiologic relationship with Epstein Barr virus. The tumor is distinctly radiosensitive, and despite the undifferentiated appearance of the tumor cells histologically, its prognosis is relatively favorable. Patients with stage I disease usually receive radiotherapy with excellent outcomes. Patients with stage II-IVB nasopharyngeal cancers are treated with concurrent chemotherapy and radiotherapy, followed by consideration for adjuvant chemotherapy. Both primary tumor and cervical metastases of nasopharyngeal carcinoma are highly sensitive to chemoradiotherapy and even bulky metastases can be successfully treated. Surgery is reserved for salvage of recurrences and persistent metastases. The surgical approach and ability to achieve negative margins for primary tumors may be difficult. However, in the circumstance of recurrent disease after radiation therapy surgery may be the only feasible therapeutic option [3].
With regard to the common variety of squamous cell carcinoma, the choice of treatment of primary tumors in the head and neck is based on both expected oncologic outcome as well as the optimal functional outcome. For resectable cancers, functional and organ preservation considerations usually guide the choice between initial surgery as opposed to (chemo)radiotherapy. In the modern era, there is a trend in treatment of locally advanced head and neck cancer to move from extensive ablative surgery with high risk of postoperative complications and loss of important functions, to non-surgical protocols of organ preservation based on concurrent chemoradiotherapy. Patients requiring a total laryngectomy or an extensive resection of the oropharynx with planned reconstruction are the most eligible candidates to enter these protocols, unless they have a poor general status or comorbidities that preclude the use of chemotherapy.

Oropharyngeal cancers, particularly those with poorly and undifferentiated carcinoma histology, are frequently associated with human papillomavirus (HPV); these tumors often exhibit strong p16 protein expression and have low rates of p53 mutations [4]. With regard to HPV status, a site-specific analysis shows that patients with HPV-positive oropharyngeal tumors have a $28 \%$ reduced risk of death in comparison to patients with HPV-negative oropharyngeal tumors. This improved survival for HPV-positive patients is specific to the oropharynx [5]. Furthermore, for patients with carcinomas of the oropharynx, tumor HPV status is strongly associated with therapeutic response and survival when treated with concurrent chemoradiotherapy [6]. Consequently, it may be reasoned that HPV-positive oropharyngeal tumors in particular can benefit from non-surgical therapy [7].

It is critical to note that the true objective of organ preservation is functional preservation and not the avoidance of surgery [8]. Thus, initial non-surgical treatment strategies may not be optimal therapy for a locally advanced laryngeal cancer that has destroyed much of that organ, implying that proper selection of patients for conservative treatment is crucial. Total laryngectomy remains the standard of care for most patients presenting with T4 disease. Moreover, appropriate partial laryngectomy procedures may also be viewed as organ and function preserving.

Generally speaking, surgery may be considered as not indicated when the chance of cure is equivalent and associated morbidity is lower with (chemo)radiotherapy. A classical example is a T1 glottic carcinoma which can be successfully treated with either radiotherapy or surgery, with similar control rates [9]. Although good voice outcomes can be obtained with endolaryngeal cordectomies with or without laser $[9,10]$, in the more extensive and deeply invasive tumors the risk of hoarseness should be discussed with the patient and treatment should be chosen in the context of the patients' needs and social activities. 
The necessity of post-chemoradiotherapy ("planned") neck dissection for patients with N2/N3 disease has been a controversial issue for a long time. With positron-emission tomography (PET) showing improved accuracy for response assessment of $\mathrm{N} 2 / \mathrm{N} 3$ disease, neck dissections can be restricted to patients who present abnormal metabolic activity on the post-treatment PET scan [11]. Another controversy is the role of elective neck dissection in the initial treatment setting, although there is consensus that elective neck treatment should not be done for tumors with a low chance (less than $20 \%$ ) of occult metastasis, as well as for histological tumor types with a low rate of regional metastasis such as adenoid cystic carcinoma. In a survey by van Weert et al. [12], only $10 \%$ of patients with adenoid cystic carcinoma presented at diagnosis with neck metastases. This low rate does not justify an elective neck dissection. Therefore, neck dissection may be considered an overtreatment unless the patient has clinically enlarged and/or cytologically confirmed nodal metastases [13].

Verrucous carcinoma is a highly differentiated variant of squamous cell carcinoma. The oral cavity is the most common site of occurrence, but in addition, it is known to occur in the larynx, pharynx and many other sites. The clinical presentation of lymphadenopathy may tempt the surgeon to perform a lymph node dissection. However, Walvekar et al. [14] observed in a series of 101 oral verrucous carcinomas, from which T3/T4 accounted for $52.4 \%$ of all tumors, and $30.7 \%$ presented with clinically positive neck nodes, that none of the patients undergoing neck dissections had pathologically positive nodes. It can be assumed that most enlarged lymph nodes at presentation are often reactive to a secondary infection or inflammation rather than metastasis [15]. Therefore, neck dissection is not indicated in pure verrucous carcinoma, unless the definitive pathological diagnosis is changed to hybrid verrucous squamous cell carcinoma. Laryngeal verrucous carcinomas behave similarly to oral verrucous carcinomas, and many authors believe that there is no metastatic potential and that neck dissection is not indicated for these tumors [16]. Furthermore, in patients with extensive laryngeal verrucous carcinoma, particularly those located in the glottic region in which surgery would cause important functional and/or cosmetic impairment, (chemo)radiotherapy could be an effective and potentially curative alternative to standard surgery [17].

Thyroid cancers behave in a characteristic manner depending on their histologic type. Papillary thyroid carcinoma (PTC) is characterized by a high incidence of occult nodal metastases. However, given the excellent prognosis of PTC, the good control of occult metastatic disease with the administration of postoperative radioiodine, and since the risks of transient nerve paralysis and hypoparathyroidism may outweigh the benefits, elective neck dissection is not indicated in the absence of nodal metastases detected either by clinical examination or ultrasound of the neck [18].

Incurable disease usually is another contraindication for surgery or any other type of aggressive treatment although surgery may be considered in certain palliative settings. A patient may be considered incurable based on factors like severe comorbidity, extensive locoregional tumor growth or distant disease.

Unresectable tumors are not always incurable, and conversely, resectable tumors are not necessarily curable. The chance of cure decreases in advanced stage disease and the decision about treatment should be based on the balance between estimated survival and the morbidity associated with the treatment, either surgical or non-surgical. There are some instances when the patient is so debilitated that any curative treatment is not possible. Some patients have very poor nutritional status or uncontrollable cardiac, hepatic or pulmonary diseases. Treatment is usually palliative for these patients unless the comorbidities can be significantly improved.

Last, the treating institution should have access to the necessary personnel, skills and equipment to perform the proposed surgery. Conversely, in some instances, surgical treatment is easier and requires fewer ancillary facilities than elaborate non-surgical protocols. This factor may have particular importance in "third world" countries.

\section{Conclusions}

In summary, the choice of treatment of malignant epithelial tumors of the head and neck generally depends on many factors and aims at an optimal oncologic outcome paired with functional outcomes and quality of life after treatment. Sometimes the choice of treatment in this perspective is difficult and several factors need to be considered and weighed; however, in some cases the choice is more obvious. In this paper, we have discussed situations in which surgical treatment is either inadvisable or contraindicated. Histological tumor type, stage and extent of disease and patient comorbidities, alone or in combination, are the usual determinants of the indication or contraindication for surgical therapy of malignant epithelial tumors of the head and neck. The available personnel and equipment may be a strong determinant of the choice between surgical and nonsurgical treatments; particularly in the developing world.

\section{References}

1. Biller HF, Lawson W, Sachdev VP, Som P (1990) Esthesioneuroblastoma: surgical treatment without radiation. Laryngoscope 100:1199-1201 
2. van der Laan TP, Bij HP, van Hemel BM et al (2013) The importance of multimodality therapy in the treatment of sinonasal neuroendocrine carcinoma. Eur Arch Otorhinolaryngol 270:2565-2568

3. Suárez C, Rodrigo JP, Rinaldo A, Langendijk JA, Shaha AR, Ferlito A (2010) Current treatment options for recurrent nasopharyngeal cancer. Eur Arch Otorhinolaryngol 267:1811-1824

4. Carpenter DH, El-Mofty SK, Lewis JS Jr (2011) Undifferentiated carcinoma of the oropharynx: a human papillomavirus-associated tumor with a favorable prognosis. Mod Pathol 24:1306-1312

5. Ragin CC, Taioli E (2007) Survival of squamous cell carcinoma of the head and neck in relation to human papillomavirus infection: review and meta-analysis. Int J Cancer 121:1813-1820

6. Fakhry C, Westra WH, Li S et al (2008) Improved survival of patients with human papillomavirus-positive head and neck squamous cell carcinoma in a prospective clinical trial. J Natl Cancer Inst 100:261-269

7. Haigentz M Jr, Silver CE, Corry J et al (2009) Current trends in initial management of oropharyngeal cancer: the declining use of open surgery. Eur Arch Otorhinolaryngol 266:1845-1855

8. Brizel DM, Esclamado R (2006) Concurrent chemoradiotherapy for locally advanced, nonmetastatic, squamous carcinoma of the head and neck: consensus, controversy, and conundrum. J Clin Oncol 24:2612-2617

9. Yoo J, Lacchetti C, Hammond JA, Gilbert RW; the Head and Neck Cancer Disease Site Group (2013) The role of endolaryngeal surgery (with or without laser) versus radiotherapy in the management of early (T1) glottic cancer: a systematic review. Head Neck. doi: 10.1002/hed.23504

10. Jamal N, Sofer E, Chhetri DK (2013) Treatment considerations for early glottic carcinoma: lessons learned and a primer for the general otolaryngologist. Otolaryngol Head Neck Surg [Epub ahead of print]

11. Hamoir M, Ferlito A, Schmitz S et al (2012) The role of neck dissection in the setting of chemoradiation therapy for head and neck squamous cell carcinoma with advanced neck disease. Oral Oncol 48:203-210

12. van Weert S, Bloemena E, van der Waal I et al (2013) Adenoid cystic carcinoma of the head and neck: a single-center analysis of 105 consecutive cases over a 30-year period. Oral Oncol 49:824-829

13. Ferlito A, Barnes L, Myers EN (1990) Neck dissection for laryngeal adenoid cystic carcinoma: is it indicated? Ann Otol Rhinol Laryngol 99:277-280

14. Walvekar RR, Chaukar DA, Deshpande MS et al (2009) Verrucous carcinoma of the oral cavity: a clinical and pathological study of 101 cases. Oral Oncol 45:47-51

15. Devaney KO, Ferlito A, Rinaldo A, El-Naggar AK, Barnes L (2011) Verrucous carcinoma (carcinoma cuniculatum) of the head and neck: what do we know now that we did not know a decade ago? Eur Arch Otorhinolaryngol 268:477-480

16. Sllamniku B, Bauer W, Painter C, Sessions D (1989) Clinical and histopathological considerations for the diagnosis and treatment of verrucous carcinoma of the larynx. Arch Otorhinolaryngol 246:126-132

17. Strojan P, Soba E, Budihna M, Auersperg M (2005) Radiochemotherapy with vinblastine, methotrexate, and bleomycin in the treatment of verrucous carcinoma of the head and neck. J Surg Oncol 92:278-283

18. Mehta V, Nathan CA (2013) Prophylactic neck dissection in papillary thyroid cancer: when is it necessary? Laryngoscope 123:2339-2340 